\section{CHEMICAL TOOLS}

\section{Pass the persulfides}

ChemBioChem doi:10.1002/cbic.201402312

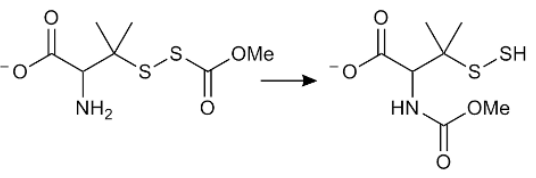

Hydrogen sulfide $\left(\mathrm{H}_{2} \mathrm{~S}\right)$ is thought to exert its biological effects through the S-sulfhydration of biological molecules, such as cysteine side chains, forming persulfides. However, studying this important modification has been challenging owing to the intrinsic reactivity of persulfides. Additionally, existing synthetic methods to generate persulfides use $\mathrm{H}_{2} \mathrm{~S}$ in reactions that typically do not proceed to completion, making it difficult to conclusively determine that biological outcomes are linked to the presence of the persulfide rather than $\mathrm{H}_{2} \mathrm{~S}$. To assist in these efforts, Artaud and Galardon envisioned a new route to make persulfides. It has been shown that acyl-protected disulfides can be converted to persulfides and that this process can be influenced by the presence of amines. The authors build on this chemistry in their design of a stable precursor molecule, $\mathrm{P}^{*}$, which contains a protected disulfide adjacent to an amine that can, when deprotonated, participate in an intermolecular acyl transfer to generate an acylated amine and persulfide group (shown). The acyl transfer occurs on a rapid timescale at near-neutral $\mathrm{pH}$ without evolving $\mathrm{H}_{2} \mathrm{~S}$. The authors successfully apply their new reagent in two cases known to involve persulfides: the sulfhydration of 8-NO2-cGMP and the reduction of cytochrome c. $\mathrm{P}^{\star}$ can be made in a single synthetic step and is stable in solid form, enabling further applications of this new tool in chemical biology research.

POST-TRANSLATIONAL MODIFICATIONS

\section{SUMO size me}

Nat. Struct. Mol. Biol. doi:10.1038/nsmb.2890

The reversible attachment of small ubiquitinlike modifier (SUMO) proteins to the lysine side chains of specific proteins is necessary for genome stability and transcription. Whereas the ubiquitination of a protein frequently leads to its degradation, SUMOylation alters the biological function of the target protein by enabling it to interact with new protein partners. Hendriks et al. have now used high-resolution MS to identify $>4,300$ SUMOylation sites in $>1,600$ proteins in human cells. Although MS has been used to identify SUMOylation sites in cells before, these authors used a new purification strategy that was able to find these sites more efficiently. Most ( $64 \%$ ) of the identified proteins had only one or two SUMO groups, but the authors identified 96 proteins that were functionalized with ten or more SUMOs. Analysis of their dataset revealed that SUMOs and other post-translational modifications (PTMs) may compete for the same lysine side chains, as $22 \%$ of SUMOylation sites were known ubiquitination sites, and SUMOylation also occurred at known acetylation and

Microglia express a number of genes that have been implicated in the pathology of Alzheimer's disease (AD), which is characterized by extracellular accumulation of amyloid- $\beta$ peptide $(A \beta)$ peptides and intraneuronal aggregates of hyperphosphorylated tau. One of these genes encodes CX3CR1, a receptor expressed exclusively by microglia that binds either a membrane-anchored or a cleaved soluble form of a neuron-generated chemokine ligand, CX3CL1. CX3CR1 knockout ameliorates disease hallmarks in an AD mouse model (called APPPS1) via enhanced $A \beta$ clearance but exacerbates tau phosphorylation and aggregation in a related tauopathy model (called $\mathrm{hTau}$ ). To understand this discrepancy and dissect the roles of the membrane-anchored versus the soluble form of CX3CL1, Lee et al. generated APPPS1 mice that expressed only the soluble CX3CL1 and found no difference in $A \beta$ deposition when compared to APPPS1 mice expressing no CX3CL1. However, when compared with CX3CL1expressing APPPS1 mice, the soluble CX3CL1-expressing mice had increased intraneuronal tau phosphorylation. CX3CL1 knockout mice had similarly elevated intraneuronal tau phosphorylation but had reduced extracellular $A \beta$ deposition. Transcriptomic experiments found three genes, encoding two inflammatory cytokines and an $A \beta$ phagocytic receptor (MSR1), that were consistently altered in microglia from the four mice strains and that correlated with the effects on $A \beta$ deposition. These results and experiments examining downstream signaling via $\mathrm{p} 38 \mathrm{MAPK}$ and $\mathrm{A} \beta$ uptake implicate the membrane-anchored CX3CL1 in the apparent opposing effects on AD pathologies via enhanced MSR1-dependent phagocytosis of $A \beta$ and decreased MAPK activation.

methylation sites. As expected, SUMOylation was found almost exclusively on nuclear or chromatin-associated proteins, with proteins involved in nucleic acid metabolism, nucleosome organization and transcription being the most common substrates. Validation of these $>3,000$ new putative SUMOylation sites should reveal how the attachment and removal of this PTM is able to alter the functions of proteins involved in so many different biological processes.

CELL CYCLE

\section{Mitotic tag team}

Nature doi:10.1038/nature13660

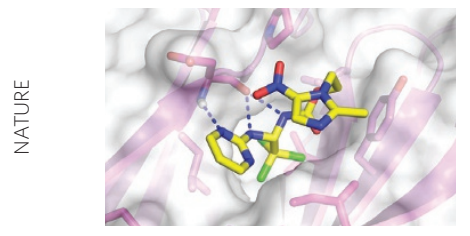

The APC/C is a multisubunit $\mathrm{E} 3$ ubiquitin ligase complex that becomes activated during mitosis through Cdc20 binding and targets $\mathrm{D}$-box-containing substrates for proteasome-mediated degradation that ends mitosis. Cdc20 binding to the D-box motif of the substrate can strengthen existing $\mathrm{APC} / \mathrm{C}$ and $\mathrm{Cdc} 20$ interactions. Tosyl-Larginine methyl ester (TAME) is a small molecule known to disrupt the interaction between APC and Cdc20 and transiently arrest cells in mitosis. Sackton et al. thought that it might be possible to cause permanent mitotic arrest and eventual cell death by disrupting APC/C-mediated interactions with substrates. One potential smallmolecule candidate was apcin, which was previously described as an inhibitor of cyclin degradation, but the identity of its targets remained unknown. The authors incubated Xenopus laevis extracts with apcin-coupled beads and found that $\mathrm{Cdc} 20$ was specifically removed from the extract, resulting in the loss of ubiquitinylation and eventual stabilization of substrates such as cyclin B1 and securin. Apcin binding to Cdc 20 could not be disrupted by the addition of TAME, suggesting distinct binding interactions for the two compounds. They determined the crystal structure of the apcin-Cdc20 complex, which revealed that apcin bound to the D-box-binding site of Cdc20, preventing access for substrate binding. The combination of apcin and TAME exhibited synergistic effects in stabilizing APC/C substrates and increased mitotic duration compared to single drug treatments, confirming that blocking multiple protein-protein interactions of a large protein machine may be an effective targeting strategy. 